Founded by H. C. Schumacher in 1821
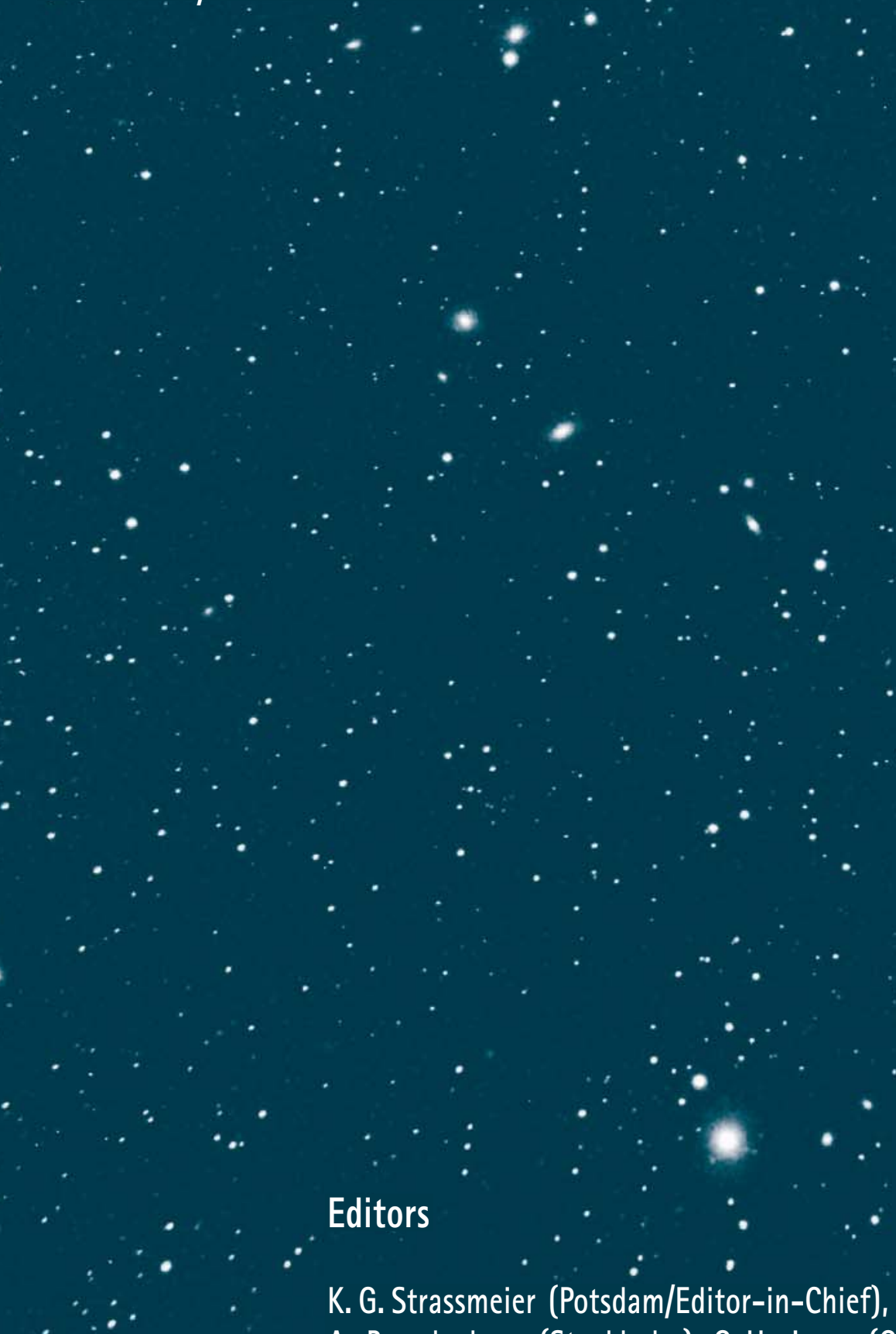

A. Brandenburg (Stockholm), G. Hasinger (Garching), R.-P. Kudritzki (Honolulu), T. Montmerle (Grenoble), H. W. Yorké (Pasạdena) 


\title{
High-energy pulsar models: Developments and new questions
}

\author{
C. Venter ${ }^{1, \star}$ and A.K. Harding ${ }^{2}$ \\ ${ }^{1}$ Centre for Space Research, North-West University, Potchefstroom Campus, Private Bag X6001, Potchefstroom 2520, \\ South Africa \\ 2 Astrophysics Science Division, NASA Goddard Space Flight Center, Greenbelt, MD 20771, USA
}

Received 2013 Dec 20, accepted 2014 Jan 14

Published online 2014 Mar 16

Key words gamma rays: stars - magnetic fields - pulsars: general - radiation mechanism: non-thermal

The past few years have seen a major advance in observational knowledge of high-energy (HE) pulsars. The Fermi Large Area Telescope (LAT) and AGILE have increased the number of known $\gamma$-ray pulsars by an order of magnitude, its members being divided roughly equally among millisecond pulsars (MSPs), young radio-loud pulsars, and young radio-quiet pulsars. Many new and diverse emission characteristics are being measured, while radio and X-ray follow-up observations increase the pulsar detection rate and enrich our multiwavelength picture of these extreme sources. The wealth of new data has provided impetus for further development and improvement of existing theoretical pulsar models. Geometric light curve (LC) modelling has uncovered three broad classes into which HE pulsars fall: those where the radio profile leads, is aligned with, or lags the $\gamma$-ray profile. For example, the original MSP and original black widow system are members of the second class, requiring co-located emission regions and thereby breaking with traditional notions of radio emission origin. These models imply narrow accelerator gaps in the outer magnetosphere, indicating copious pair production even in MSP magnetospheres that were previously thought to be pair-starved. The increased quality and variety of the LCs necessitate construction of ever more sophisticated models. We will review progress in global magnetosphere solutions which specify a finite conductivity on field lines above the stellar surface, filling the gap between the standard vacuum and force-free (FF; plasma-filled) models. The possibility of deriving phase-resolved spectra for the brightest pulsars, coupled with the fact that the HE pulsar population is sizable enough to allow sampling of various pulsar geometries, will enable much more stringent testing of future radiation models. Reproduction of the observed phase-resolved behavior of this disparate group will be one of the next frontiers in pulsar science, impacting on our understanding of particle acceleration, emission, and magnetosphere geometry. One may now also study evolutionary trends of the measured or inferred quantities, and probe pulsar visibility and population properties such as radiation beam sizes of different pulsar classes, as well as the distribution of spin-down power, $\gamma$-ray luminosity, conversion efficiency, spectral index, and cutoff energy across the population. Lastly, the recent detection of very-high-energy (VHE) pulsations from the Crab pulsar generated quite a few ideas to explain this emission, leading to an extension of standard models and possibly even a bridge between the physics of pulsars and pulsar wind nebulae (PWNe).

(c) 2014 WILEY-VCH Verlag GmbH \& Co. KGaA, Weinheim

\section{Introduction}

The incredible rate of $\gamma$-ray pulsar detections by the Fermi Large Area Telescope (LAT) in the GeV band has brought tremendous progress to this field. The Second Pulsar Catalog (2PC; Abdo et al. 2013) details the properties of 117 high-confidence detections above $100 \mathrm{MeV}$ using 3 years of Fermi data, with $50 \%$ of these pulsars having been discovered through $\gamma$-ray pulsations or by radio searches of $\gamma$-ray sources. The population is distributed evenly among millisecond pulsars (MSPs), young radio-loud pulsars, and young radio-quiet pulsars. For a discussion of their multiband profiles, off-peak emission properties, luminosities, spectra, and other population trends, see $2 \mathrm{PC}$. Radio and Xray follow-up observations have also played an important role in many of these detections, giving multiwavelength constraints on source energetics and viewing geometry.

\footnotetext{
^ Corresponding author: Christo.Venter@nwu.ac.za
}

Many novel emission characteristics have come to light, stimulating a number of theoretical developments. We will summarize results from geometric light curve (LC) modelling (Sect. 2), focusing on both standard and new emission geometries and magnetospheric structures. Next, we will review results obtained from phase-resolved spectroscopy of the brightest $\gamma$-ray pulsars (Sect. 3), and describe results of and prospects for $\mathrm{TeV}$-domain pulsar science (Sect. 4). We lastly list some open questions in Sect. 5.

\section{Probing the magnetospheric and emission geometry via light curve modelling}

The availability of high quality $\gamma$-ray LCs has sparked a barrage of geometric LC modelling that attempts to inform us about the geometric properties of the magnetospheric emission regions. This is a necessary first step that will guide the development of more physical radiation models. Below, 
we will first describe work using the standard models, for both young and old pulsars, before turning to newer emission geometries and magnetosphere solutions.

\subsection{Standard emission geometries}

Some years after the solution by Deutsch (1955) of a magnetic field rotating in vacuum (vacuum retarded dipole; VRD), Goldreich \& Julian (1969) showed that the induced electric force vastly exceeds the gravitational pull on surface charges, so that the pulsar magnetosphere should be plasmafilled or "force-free" (FF; Spitkovsky 2006) rather than being a vacuum. However, in a fully FF magnetosphere, the accelerating electric field (parallel to the magnetic field) is screened everywhere, and there can be no pulsed nonthermal emission. There should therefore be local deviations from this ideal assumption. This led to the development of a number of physical gap models.

The polar cap (PC) model (Daugherty \& Harding 1996) assumes charge injection from the PC, with radiation taking place only at low altitudes (a few stellar radii) until secondary electron-positron pairs created from high-energy (HE) emission by relativistic primary particles screen the electric field at the so-called pair formation front. Taking the boundary condition of a zero electric field on the last open field line boundary properly into account leads to the pair formation front rising in altitude close to this boundary, forming a slot gap (SG) structure (Arons 1983; Muslimov \& Harding 2003; Muslimov \& Harding 2004a) in which particles can be accelerated and emit radiation from the surface up to the light cylinder $R_{\mathrm{LC}}$ (where the corotation speed equals the speed of light). The PC and SG models assume space-charge-limited flow, where charges are freely pulled from the stellar surface. The geometric two-pole caustic model (TPC; Dyks \& Rudak 2003) resembles this SG structure, with both magnetic poles being visible. The outer gap (OG) model (Cheng et al. 1986; Romani 1996) posits outermagnetospheric gaps reaching from the null charge surface (NCS) where the Goldreich-Julian charge density is zero up to $R_{\mathrm{LC}}$. This represents a one-pole caustic model. 'Caustic' here refers to the bunching in phase of photons radiated on trailing magnetic field lines from a range of altitudes, due to the combination of time-of-flight delays, aberration, and magnetic field line sweepback (Morini 1983; Romani \& Yadigaroglu 1995; Dyks \& Rudak 2003). This is a feature of the standard outer-magnetospheric gap models, and is key to forming the $\gamma$-ray LC peaks. Additionally, when the voltage and magnetic field are too low to facilitate pair formation, particles may be accelerated in an unscreened open volume above the PC in the pair-starved polar cap (PSPC) model (Muslimov \& Harding 2004b; Muslimov \& Harding 2009).

\subsubsection{Younger pulsars}

Early on in the Fermi mission, Watters et al. (2009) provided a practical visual summary of the beaming properties of $\gamma$-ray pulsars. Using PC, low-altitude TPC, and OG geometries, they provided maps indicating $\gamma$-ray peak multiplicity and separation $\Delta$ as functions of the inclination and observer angles $\alpha$ and $\zeta$, and gap width $w$; and also separating radio-loud and radio-quiet pulsars. Their "atlases" may be used in two ways: for a known pulsar geometry $(\alpha, \zeta)$ from multiwavelength constraints, the $\gamma$-ray pulse properties may be inferred for each of the different geometric models. Conversely, given some observed HE pulse properties, the viewing geometry may be constrained, and one may sometimes discriminate between different geometric models. Watters et al. (2009) noted that a plot of $\Delta$ vs. radio-to- $\gamma$ phase lag $\delta$ (the " $\Delta-\delta$ relation") may be a powerful discriminator between models, also constraining the radio emission properties. Lastly, they demonstrated the role of having accurate assessments of the correction factor $f_{\Omega}$, used when converting from the observed energy flux to intrinsic HE luminosity $L_{\gamma}$, as the $\gamma$-ray efficiency $\eta=L_{\gamma} / \dot{E} \propto f_{\Omega}$ (with $\dot{E}$ the spin-down power).

Romani \& Watters (2010) used Fermi LCs of 5 bright, younger pulsars, in addition to external multiwavelength constraints on the pulsar geometry (from X-ray pulsar wind nebula (PWN) torus fitting and radio polarization measurements), to constrain both the emission zone geometry and the magnetospheric structure. Using low-altitude TPC and OG geometries, as well as static, VRD, and pseudo-forcefree (PFF) magnetic fields (the latter being constructed by adding a current-induced perturbation field to the VRD), they found that the OG model and PFF magnetosphere were statistically favored. Similarly, but following a different method, Li \& Zhang (2011) used the VRD plus a perturbed field (see Muslimov \& Harding 2009) to study the effect of the latter on the LCs of the Vela pulsar, finding that a negative perturbation amplitude seems to be preferred.

Pierbattista et al. (2014) used PC, SG, and two OG geometries, in addition to conal and core radio models, in a VRD magnetosphere to model the LCs of 76 young and middle-aged LAT pulsars from 2PC. The bulk of the LCs favor outer-magnetospheric models (and high $\alpha$ and $\zeta$ ), although no single model best fits all LCs. PCs models favor low $\alpha$ and $\zeta$. Inclusion of radio LC fits as opposed to fitting $\gamma$-ray profiles only leads to solutions closer to the diagonal on an $\alpha$ vs. $\zeta$ plot, as required by radio visibility, so $\alpha$ and $\zeta$ may otherwise be underestimated in the OG models. The values for $f_{\Omega}$ are low for the PC model (given its narrowbeam geometry), $f_{\Omega} \sim 1$ for the $\mathrm{SG}$, and $f_{\Omega} \sim 0.5-0.9$ for the OG models (with radio-quiet pulsars generally requiring smaller values). Within errors, $L_{\gamma} \propto \dot{E}^{1 / 2}$. There is less scatter for the modelled $L_{\gamma}$ vs. $\dot{E}$ trend using the inferred $f_{\Omega}$ than in $2 \mathrm{PC}$ (using $f_{\Omega}=1$ ), so that this value may be an overestimation, especially for low- $\dot{E}$ objects. There is no evidence for evolution of $\alpha$ with age. Lastly, tentative inverse trends for the $\gamma$-ray cutoff energy and spectral index vs. $w$ were observed. 


\subsubsection{Millisecond pulsars}

Venter et al. (2009) geometrically modelled the LCs of the first 8 Fermi-detected MSPs using standard 3D PSPC, $\mathrm{TPC}^{1}$, and $\mathrm{OG}$ models (and the radio cone model of Story et al. 2007). Since the bulk were best fit by TPC and OG models, copious pair production must be taking place to set up the narrow emission gaps, even in MSP magnetospheres that were previously thought to be pair-starved. Venter et al. (2009) also found $f_{\Omega} \sim 1$, similar to Watters et al. (2009). Venter et al. (2012) first noted that $\gamma$-ray pulsar LCs may be categorized into three broad classes (Class I to III): those where the radio leads the $\gamma$-ray profile (fit by standard OG / TPC models), is phase-aligned with it, or lags the $\gamma$-ray peaks (exclusively fit by PSPC models). They developed two types of models for Class II pulsars, using co-located radio and $\gamma$-ray emission regions: altitude-limited TPC and OG (alTPC, alOG) models where the longitudinal gap extent and position were varied given some restrictions, and low-altitude SG (laSG) models resembling hollow cones. LC fits favored the altitude-limited models, implying caustic origin of both the $\gamma$-ray and radio emission (Guillemot et al. 2012). The radio should be associated with depolarization and rapid position angle swings (Dyks et al. 2004).

Espinoza et al. (2013) introduced a different LC classification scheme after studying the properties of $30 \gamma$-ray MSPs: (i) A-type MSPs exhibit phase-aligned radio and $\gamma$ ray peaks (equivalent to Class II MSPs; these also exhibit the largest magnetic fields at $R_{\mathrm{LC}}$, steepest radio spectra, giant radio pulses in some cases, and low radio linear polarization which is possibly indicative of caustic radio emission); (ii) N-type MSPs have non-aligned main radio and $\gamma$-ray peaks, usually with single radio peaks; (iii) the main $\gamma$-ray peak of W-type MSPs is out of phase with the main radio peak of a profile which consists of multiple peaks covering a large phase range ("wide" peaks), possibly indicating small $\alpha$. The type $\mathrm{N}$ and $\mathrm{W}$ definitions intersect with the Class I and III designations discussed earlier.

Johnson et al. (2014) systematically modelled the 40 MSPs contained in 2PC using OG, TPC, laSG, and PSPC models, combined with a semi-empirical conal radio model (Story et al. 2007), invoking core components when indicated by polarization measurements, and assuming a VRD field. They found that no single model best fits all LCs, although OG and TPC models provided best fits for the bulk of the MSP population (each best fits $\sim 40 \%$ of the MSPs). LCs with significant off-peak $\gamma$-ray emission are better fit by the TPC geometry, while LCs with little or no off-peak emission favour the OG geometry. Seven Class III MSPs are exclusively best fit by the PSPC model. This "mix" of results may point to some hybrid geometry that incorporates elements of the different standard models. The different LC classes pointed out by Venter et al. (2012) are not easily distinguished based on canonical pulsar vari-

\footnotetext{
1 The "standard" or "conventional" TPC model assumes a maximum cylindrical radius of $\rho_{\text {cyl }}=0.95 R_{\mathrm{LC}}$ for the emission region, while the "original" or "low-altitude" TPC assumes $\rho_{\text {cyl }}=0.75 R_{\mathrm{LC}}$.
}

ables (such as spin period, magnetic field, or $\dot{E}$ ) alone, but may rather reflect more complex electrodynamical environments for the different pulsars. The best-fit $\alpha$ occurred over a broad range of values, which may be a result of their spinup evolution and may also reflect the relatively wider radio beams and smaller magnetospheres of MSPs, making them visible over a larger region of phase space than their younger counterparts. This implies that there are probably not many radio-quiet MSPs. A clustering at large $\zeta$ is observed, ascribed to the bright caustic emission lying near the spin equator which is usually sampled to form the HE peaks. A preponderance of $\zeta \sim 90^{\circ}$ is expected if pulsar spin axes are distributed randomly with respect to the Earth line of sight. OG models prefer larger $\alpha$, since these gaps may be invisible for small $\alpha$ (depending on $w$ ). For MSPs, $L_{\gamma} \propto \dot{E}$, confirming the expectation that older pulsars' magnetospheres are more pair-starved, enabling them to reach relatively larger $\eta \sim 10 \%$.

\subsection{Other magnetospheres or emission geometries}

\subsubsection{TPC / OG models in an FF magnetosphere}

While numerous geometric LC studies have been done using the VRD as the basic magnetospheric structure (Sect. 2.1), the FF magnetic structure should be more realistic in that it takes into account the effect of charges and currents. The FF magnetosphere satisfies $\rho \boldsymbol{E}+\boldsymbol{j} \times \boldsymbol{B} / c=0$, with $\rho$ and $\boldsymbol{j}$ the charge and current densities, such that $\boldsymbol{E} \cdot \boldsymbol{B}=0$, implying that particle acceleration or emission is precluded (see, e.g., Contopoulos et al. 1999; Gruzinov 2005; Komissarov 2006; McKinney 2006; Spitkovsky 2006; Timokhin 2006; Kalapotharakos et al. 2012a).

Bai \& Spitkovsky (2010) presented model LCs using the conventional TPC and OG models, but in an FF magnetosphere. They assumed constant emissivity along particle trajectories in the lab frame and found that the FF TPC model cannot produce sharp double-peaked LCs due to the increased PC size compared to that of the VRD. This suppresses caustic formation close to the stellar surface, so that all caustics are produced close to $R_{\mathrm{LC}}$. For the $\mathrm{OG}$ case, emission is gathered only from open field lines that cross the NCS, and this generally eliminates one peak from the resulting LC. Next, Bai \& Spitkovsky (2010) investigated a "separatrix layer model" in the FF magnetosphere (very similar to the FF TPC, but slightly interior to it) where $\gamma$ ray emission originates in a thin layer in the open-field-line region just inside the separatrix (current sheet) which separates open and closed field lines. Field lines with footpoints close but interior to the PC rim are involved, and emission extends from the stellar surface to beyond $R_{\mathrm{LC}}$. A new caustic (one from each pole) appears which is due to "sky map stagnation" (SMS), where emission originating close to $R_{\mathrm{LC}}$ arrives at the same observer phase. Double-peaked LCs (mostly at large $\alpha$ and $\zeta$ ) are a generic feature due to caustics from both poles that form in the outer magnetosphere, where the field lines approach a split-monopolar geometry. The SMS effect becomes more pronounced as 
one chooses radiating field lines closer to the magnetic axis. $^{2}$

Harding et al. (2011) modelled TPC LCs in VRD and FF magnetospheres, investigating the effect of an azimuthal modulation in emissivity, since the PCs in these magnetospheres are offset from the magnetic axis, inducing an azimuthally asymmetric electric field (Harding \& Muslimov 2011). LCs incorporating this modulation exhibit much lower off-peak emission. The FF LC peaks are shifted later in phase (vs. VRD LCs), since the FF PCs are larger and the field lines are more swept back. They found that the Vela LCs favor the asymmetric VRD LCs, possibly indicating that the real pulsar magnetosphere solution is closer to the VRD than the FF geometry. DeCesar et al. (2014) obtained best-fit TPC and OG LCs for four bright pulsars (Vela, Crab, Geminga, and PSR J0007+7303), using VDR and FF fields. No single emission model or magnetic geometry was preferred, pointing to yet more complex emission geometries.

\subsubsection{Annular gap model}

Qiao et al. (2004) proposed an annular gap model to explain pulsar emission. On the stellar surface, an annulus is formed by the footpoints of the last open field lines (PC rim) and those of the critical field lines which intersect with the NCS at $R_{\mathrm{LC}}$. This bounded region is referred to as the annular gap (AG), while the region interior to the $A G$ is called the core gap (CG). The annulus shape is a strong function of $\alpha$ (assuming a dipolar magnetic field), and may be a source of relativistic particles that radiate between the AG and NCS.

Du et al. (2010) presented geometric HE LC modelling (no radio modelling) using a single-pole, intermediateheight 3D AG (favoring short period-pulsars). They assumed two Gaussian distributions for the emissivity: one along the field lines (peaking near the NCS), and one across the gap in the latitudinal direction (peaking near the AG center). By including aberration and retardation and also taking $\zeta$ constraints from PWN torus fitting (Ng \& Romani 2008) into account, they obtained reasonable fits for the LCs of 6 Fermi pulsars, noting that their model emission from younger pulsars peaked near the NCS, while it originated from much lower altitudes for MSPs (implying smaller beam solid angles $\Delta \Omega$ ). For Vela, Du et al. (2011) invoked $\mathrm{HE}$ emission from the AG to fit the two main peaks (P1 and P2), while the third "bump" (P3) was fit using the CG. They fit multiband LCs by adjusting the dominant emission height for each energy band. Radio emission was assumed to come from a narrow region near $R_{\mathrm{LC}}$. Using a $2 \mathrm{D}$ global electric field, they calculated phase-resolved spectra assuming synchro-curvature radiation (synchro-CR) from primary particles and synchrotron radiation (SR) from secondaries, assuming power-law spectra and adjusting $\Delta \Omega$. There are many free parameters in this approach.

\footnotetext{
2 See also Contopoulos \& Kalapotharakos (2010) who studied signatures in LCs and polarization profiles of CR originating in the strong current sheet that develops at the tip of the pulsar closed line region using time-dependent 3D FF magnetosphere.
}

Du et al. (2012) next modelled the radio-to-TeV emission from the Crab pulsar in seven phase bands (plus phaseaveraged) using the single-pole AG model. They used a 1D accelerating potential along the field lines, as well as powerlaw distributed primaries and two populations of pairs, induced by CR and inverse Compton scattering (ICS) respectively. SR from pairs dominated the X-ray to soft $\gamma$-ray band; CR and SR from primaries the $\gamma$-ray band; and ICS from pairs (on thermal surface and SR photons) the $\mathrm{TeV}$ band. In order to constrain the emission geometry and locations, they used prior geometric LC fitting invoking the AG to model the peaks, and the CG to model the bridge emission. Du et al. (2013) modelled the radio and $\gamma$-ray LCs of three MSPs (PSR J0034-0534, PSR J0101-6422, and PSR J0437-4715) using a single-pole AG plus CG model. The challenging three-peaked $\gamma$-ray LC of PSR J0101-6422 could be fit invoking emission from both the AG and two zones in the $\mathrm{CG}$, coming from different emission altitudes.

\subsubsection{Striped wind model}

Building on previous work (Coroniti 1990; Michel 1994), Pétri (2009) considered the striped wind as an alternative emission region outside $R_{\mathrm{LC}}$ to generate HE emission. He used an asymptotic oblique split monopole solution (Bogovalov 1999), noting that relativistic beaming effects give rise to pulsed emission from this region. Calculating ICS from an electron-positron pair wind on cosmic microwave background photons, Pétri could reproduce the LCs and phase-resolved spectra of Geminga using EGRET data. Pétri (2011) presented calculations of LCs for Fermi pulsars (neglecting energy dependence), also including radio emission from the PCs, modeled as a Gaussian emissivity profile peaking on the magnetic axis. Pétri noted the analytic relation $\cos (\pi \Delta)=|\cot \zeta \cot \alpha|$, first found by Kirk (2005), as well as an expression for the $\Delta-\delta$ relation $(\delta \approx 0.5-0.5 \Delta)$, interpreting the phase lag $\delta$ as a time-of-flight effect. Two regions of roughly constant $f_{\Omega}(\alpha, \zeta) \approx\{0.4,1.9\}$ were obtained. The $\delta$ in this model are generally larger than seen in the data. Another application considers $\gamma$-ray SR from a relativistically hot current sheet in the striped wind, including some magnetic reconnection (Pétri 2012).

\subsubsection{Dissipative magnetospheres}

Newer global magnetosphere solutions specify a (constant) finite conductivity $\sigma$ on field lines above the stellar surface $(\boldsymbol{E} \cdot \boldsymbol{B} \neq 0$ so that there may be particle acceleration), thereby filling the gap between the vacuum and FF models; see Kalapotharakos et al. (2012b) and Li et al. (2012). Kalapotharakos et al. (2012b) considered an orthogonal rotator, and followed two approaches to calculate HE LCs: the usual geometric approach (Sect. 2.1), and a particle trajectory approach (since in these magnetospheres, particles do not follow the magnetic field lines in the corotating frame) similar to that used in the separatrix model (Sect. 2.2.1). For larger $\sigma$ (larger field sweepback), there is a progressive 
LC shift to larger phases and peak broadening. For the second approach, narrowest pulses are found for high $\sigma$, and $\delta$ generally decreases with $\zeta$ for lower $\sigma$ values. When exclusively considering emission close to $R_{\mathrm{LC}}$ in these magnetospheres, improved predictions for the $\Delta-\delta$ trend may be obtained (Kalapotharakos et al. 2014).

\section{Phase-resolved spectroscopy}

The vast number of accumulated statistics from the brightest $\gamma$-ray pulsars is now allowing us to probe the emission environment and processes on a much deeper level than just using LC modelling. For example, Abdo et al. (2010) presented phase-resolved spectra of the Vela pulsar, showing the variation of photon index and cutoff energy $E_{\mathrm{c}}$ as a function of phase $\phi$. Although this has been done previously (e.g., Kanbach et al. 1994; Fierro et al. 1998), much finer phase bins may now be attained and more complicated spectral shapes may be fit to data in a particular phase bin.

DeCesar et al. (2014) studied four bright Fermi pulsars using 30 months of data. The LC peaks are thought to be due to caustic emission (Sect. 2.1), with photons originating from very different emission radii being contained in any particular phase bin. Therefore, the inferred $E_{\mathrm{c}}$ reflects the combination of emitted spectra from various positions in the magnetosphere (sampling different local conditions, e.g., magnetic field and curvature radius $\rho_{\mathrm{c}}$ ). This complicates the disentanglement of altitudinal behavior of the accelerating electric field $E_{\|}(r)$ when one only finds an indication of the combined effect of many different local $E_{||}$and $\rho_{\mathrm{c}}$ contributing to the spectral shape at a particular phase $\phi$ through the measured $E_{\mathrm{c}}(\phi)$. Nevertheless, when assuming that $\mathrm{CR}$ is the dominant emission process in the $\mathrm{GeV}$ band and that the $\mathrm{CR}$ reaction limit is valid, one may constrain the implied $E_{\|}(\phi)$ using the measured $E_{\mathrm{c}}(\phi)$ and modelled $\rho_{\mathrm{c}}^{\max }(\phi)$, and $\rho_{\mathrm{c}}^{\min }(\phi)$ for a particular inferred pulsar geometry (from the LC modelling) and magnetosphere, and finally infer $E_{\|}\left(r_{\min }\right)$ using the modelled minimum emission radius $r_{\min }(\phi)$. This in turn constrains the magnetospheric structure, implying that high-altitude field lines should be straighter (higher $\rho_{\mathrm{c}}$ ) compared to the vacuum case if $E_{\|}$is not to exceed the local magnetic field (as it should be, since it is induced by this magnetic field in the first place). The grand challenge for future physical emission models will be to reproduce both the energy-dependent LCs as well as the phase-dependent spectral behavior seen in a population of bright $\gamma$-ray pulsars, each having unique geometries, as this will yield the most stringent constraints to date on particle acceleration, emission, and magnetic field geometry in the pulsar magnetosphere.

\section{Very-high-energy pulsar science}

Many of the Fermi-detected pulsars exhibit exponentially cutoff spectra (2PC). This has been interpreted as CR by relativistic particles moving along curved field lines. Typical pulsar parameters result in cutoffs around a few $\mathrm{GeV}$, exactly what Fermi has seen (e.g., Venter \& de Jager 2010). While earlier models predicted that these cutoffs may reach $100 \mathrm{GeV}$ (Bulik et al. 2000; Harding et al. 2002) in MSPs, later PSPC MSP models predicted lower cutoffs $\sim 10$ $50 \mathrm{GeV}$ (Harding et al. 2005; Venter \& de Jager 2005; Frackowiak \& Rudak 2005). The SG model applied to the Crab pulsar predicted cutoffs up to a few GeV (Harding et al. 2008), while the OG model could explain $\gamma$-rays of up to $25 \mathrm{GeV}$ (e.g., Hirotani 2008; Tang et al. 2008). Given this theoretical context, it was very surprising to detect pulsed emission at tens to hundreds of $\mathrm{GeV}$ from the Crab pulsar (Aliu et al. 2008, Saito 2010, Aleksiç et al. 2011, Aliu et al. 2011, Aleksiç et al. 2012), indicating a necessary extension of the standard pulsar models.

Spectral cutoffs are determined by the maximal particle acceleration, as well as (one- and two-photon) absorption of energetic $\gamma$-rays (e.g., Aliu et al. 2008). One needs an unrealistically high value for the accelerating electric field $\left(E_{\|} \sim B\right)$ and/or radius of curvature $\left(\rho_{\mathrm{c}} \sim R_{\mathrm{LC}}\right)$ to explain the observed VHE emission when invoking CR with a spectral cutoff or break around $100 \mathrm{GeV}$ (Aliu et al. 2011).

A revised OG model by Hirotani may produce inverse Compton (IC) radiation of up to $400 \mathrm{GeV}$ by secondary and tertiary pairs on infrared to ultraviolet (UV) photons to explain the observed Crab spectrum (Aleksiç et al. 2012). Extending the calculations within an SG model, assuming non-isotropic IC of SR photons by the pair plasma, may prove useful. This approach is however very dependent on the assumed electrodynamics and magnetic field geometry, making the spectral prediction somewhat uncertain. Lyutikov et al. (2012) proposed a synchrotron self-Compton model to explain the VHE emission. They assume that the electron-positron plasma upscatter UV SR photons in the Klein-Nishina regime and obtain a reasonable reproduction of the pulsed VHE spectrum. They also argue for a statistical correlation between X-ray and $\gamma$-ray photons on a short timescale. The difference in HE and VHE LCs, and the flattening of the spectrum with energy motivated Aharonian et al. (2012) to invoke a low-energy, mono-energetic, ultrarelativistic wind dominated by kinetic particle energy that is very rapidly accelerated close to $R_{\mathrm{LC}}$ to upscatter (mainly X-ray) photons from the pulsar surface and/or magnetosphere via anisotropic IC scattering. Other possibilities beyond extensions to the standard scenarios include the AG model by Du et al. (2012), or SR by primary electrons with very high Lorentz factors $\left(\gamma \sim 10^{9}\right.$; Chkheidze et al. 2011).

\section{Conclusion}

With the availability of high-quality $\gamma$-ray data the emphasis has now shifted to model refinement, entailing a move beyond the standard vacuum-based (or even FF) magnetosphere models toward self-consistent, dissipative magnetospheres that determine both the geometry and position- 
dependent emissivity of the emitting region from first principles. The question of choosing a best model should therefore change from a "TPC vs. OG argument" to constraints on the plasma properties (e.g., density and conductivity), also taking into account the effect of pulsar evolution. We are thus moving toward constraining the actual pulsar electrodynamics, and not just geometry. However, integrating a physics-motivated prescription for the conductivity (the microphysics of pair cascades) into the dissipative magnetosphere models is a necessary next step. Lastly, a population synthesis approach (e.g., Watters \& Romani 2011; Takata et al. 2011; Pierbattista et al. 2012) which includes more realistic magnetospheres and emission geometries should be a key avenue for future progress.

We conclude this review by listing some open questions:

1. There is a rich variety of LCs in the newly-detected $\gamma$-ray pulsar population; is this solely due to geometry, or may distinct magnetospheric conditions and evolution also play a role?

2. There is no overall best-fit geometrical LC model; this points to some hybrid or new emission geometry. What would such a geometry be?

3. Preliminary trends are seen between geometric/ spectral vs. pulsar parameters. Will these trends strengthen with more pulsar discoveries, or do we need to study a larger phase space, including noncanonical pulsar parameters?

4. Which is the preferred magnetospheric structure? What is the effect of the current sheet? Does magnetic reconnection play a role to dissipate particle energy and create emission?

5. The behavior of spectral parameters with phase is highly complex; how is this explained? Will similar behavior be seen when phase-resolved spectroscopy of dimmer pulsars becomes viable?

6. What is the origin of pulsed $\mathrm{TeV}$ photons from pulsars?

Acknowledgements. CV is supported by the South African National Research Foundation. AKH acknowledges support from the NASA Astrophysics Theory Program. CV and AKH acknowledge support from the Fermi Guest Investigator Program.

\section{References}

Abdo, A.A., et al. 2010, ApJ, 713, 154

Abdo, A.A., et al. 2013, ApJS, 208, 17 (2PC)

Aharonian, F.A., et al. 2012, Nature, 482, 507

Aleksiç, J., et al. 2011, ApJ, 742, 43

Aleksiç, J., et al. 2012, A\&A, 540, A69

Aliu, E., et al. 2008, Science, 322, 1221

Aliu, E., et al. 2011, Science, 334, 69

Arons, J. 1983, ApJ, 266, 215

Bai, X.-N., \& Spitkovsky, A. 2010, ApJ, 715, 1282

Bogovalov, S.V. 1999, A\&A, 349, 1017

Bulik, T., Rudak, B., \& Dyks, J. 2000, MNRAS, 317, 97

Cheng, K.S., Ho, C., \& Ruderman, M. 1986, ApJ, 300, 500

Chkheidze, N., et al. 2011, ApJ, 730, 62

Contopoulos, I., Kazanas, D., \& Fendt, C. 1999, ApJ, 511, 351

Contopoulos, I., \& Kalapotharakos, C. 2010, MNRAS, 404, 767

Coroniti, F.V. 1990, ApJ, 349, 538
Daugherty, J.K., \& Harding, A.K. 1996, ApJ, 458, 278

DeCesar, M.E., et al. 2014, in preparation

Deutsch, A.J. 1955, Ann. d'Astrophys., 18, 1

Du, Y.J., et al. 2010, MNRAS, 406, 2671

Du, Y.J., Han, J.L., Qiao, G.J., \& Chou, C.K. 2011, ApJ, 731, 2

Du, Y.J., Qiao, G.J., \& Wang, W. 2012, ApJ, 748, 84

Du, Y.J., Qiao, G.J., \& Chen, D. 2013, ApJ, 763, 29

Dyks, J., \& Rudak, B. 2003, ApJ, 598, 1201

Dyks J., Harding, A.K., \& Rudak, B. 2004, ApJ, 606, 1125

Espinoza, C.M., et al. 2013, MNRAS, 430, 571

Fierro, J.M., et al. 1998, ApJ, 494, 734

Frạckowiak, M., \& Rudak, B. 2005, Adv. Space Res., 35, 1152

Goldreich, P., \& Julian, W.H. 1969, ApJ, 157, 869

Gruzinov, A. 2005, Phys. Rev. Lett., 94, 021101

Guillemot, L., et al. 2012, ApJ, 744, 33

Harding, A.K., Muslimov, A.G., \& Zhang, B. 2002, ApJ, 576, 366

Harding, A.K., Usov, V.V., \& Muslimov, A.G. 2005, ApJ, 622, 531

Harding, A.K., Stern, J.V., Dyks, J., \& Frạckowiak, M. 2008, ApJ, 680,1378

Harding, A.K., DeCesar, M.E., Miller, M.C., Kalapotharakos, C., \& Contopoulos, I. 2011 Fermi Symp. Proc., eConf C110509

Harding, A.K., \& Muslimov, A.G. 2011, ApJ, 743, 181

Hirotani, K. 2008, ApJ, 688, L25

Johnson, T.J., et al. 2014, in preparation

Kalapotharakos, C., Contopoulos, I., \& Kazanas, D. 2012a, MNRAS, 420, 2793

Kalapotharakos, C., et al. 2012b, ApJ, 754, L1

Kalapotharakos, C., et al. 2014, in preparation

Kanbach, G., et al. 1994, A\&A, 289, 855

Kirk, J.G. 2005, Mem. Soc. Astron. Ital., 76, 494

Komissarov, S.S. 2006, MNRAS, 367, 19

Li, X., \& Zhang, L. 2011, ApJ, 743, 113

Li, J., Spitkovsky, A., \& Tchekhovskoy, A. 2012, ApJ, 746, 60

Lyutikov, M., Otte, N., \& McCann, A. 2012, ApJ, 754, 33

McKinney, J.C. 2006, MNRAS, 368, L30

Michel, F.C. 1994, ApJ, 431, 397

Morini, M. 1983, MNRAS, 202, 495

Muslimov, A.G., \& Harding, A.K. 2003, ApJ, 588, 430

Muslimov, A.G., \& Harding, A.K. 2004a, ApJ, 606, 1143

Muslimov, A.G., \& Harding, A.K. 2004b, ApJ, 617, 471

Muslimov, A.G., \& Harding, A.K. 2009, ApJ, 692, 140

Ng, C.-Y., \& Romani, R.W. 2008, ApJ, 673, 411

Pétri, J. 2009, A\&A, 503, 13

Pétri, J. 2011, MNRAS, 412, 1870

Pétri, J. 2012, MNRAS, 424, 2023

Pierbattista, M., et al. 2012, A\&A, 545, A42

Pierbattista, M., et al. 2014, ApJ, in preparation

Qiao, G.J., et al. 2004, ApJ, 606, L49

Romani, R.W. 1996, ApJ, 470, 469

Romani, R.W., \& Watters, K.P. 2010, ApJ, 714, 810

Romani, R.W., \& Yadigaroglu, I.-A. 1995, ApJ, 438, 314

Saito, T. 2010, $\mathrm{PhD}$ thesis, Ludwig-Maximilians-Universität München, Germany

Spitkovsky, A. 2006, ApJ, 648, L51

Story, S.A., Gonthier, P.L., \& Harding, A.K. 2007, ApJ, 671, 713

Takata, J., Wang, Y., \& Cheng, K.S. 2011, ApJ, 726, 44

Tang, A.P.S., et al. 2008, ApJ, 676, 562

Timokhin, A.N. 2006, MNRAS, 368, 1055

Venter, C., \& de Jager, O.C. 2005, ApJ, 619, L167

Venter, C., Harding, A.K., \& Guillemot, L. 2009, ApJ, 707, 800

Venter, C., \& de Jager, O.C. 2010, ApJ, 725, 1903

Venter, C., Johnson, T.J., \& Harding, A.K. 2012, ApJ, 744, 34

Watters, K.P., et al. 2009, ApJ, 695, 1289

Watters, K.P., \& Romani, R.W. 2011, ApJ, 727, 123 